\title{
A study of sub-basalt depth imaging using the local Radon attributes on the Erlend Tertiary volcanic complex - North of Shetland, UK
}

\author{
A. Droujinine ${ }^{1, *}$, J. Pajchel ${ }^{2} \&$ K. Hitchen ${ }^{1}$
}

1 British Geological Survey, West Mains Road, Edinburgh EH9 3LA UK.

2 Norsk Hydro Research Centre, P.0. Box 7190, Bergen 5020, Norway.

* Corresponding author. Present address: Shell International E\&P B.V., Kessler Park 1, 2288 GS Rijswijk-ZH, the Netherlands. Email: Alexander.Droujinine@shell.com

Manuscript received: May 2007; accepted: December 2007

\begin{abstract}
Acquiring conventional $3 \mathrm{~km}$ towed streamer data along a 2D profile in the North of Shetland (UK) enables us to use the local Radon-attributes within the context of depth processing methodology for accurate delineation of volcanic units and imaging beneath high-velocity layers. The objective is to map the radially-dipping structure of the Erlend pluton and to investigate the potential existence of relatively soft Cretaceous sediments underneath volcanic units. Success in the Erlend Volcano study requires strict attention to the separation between different groups of events. The crucial point is the generalized discrete Radon transform formulated in terms of local wavefront (dip and curvature) characteristics. This transform is utilized during pre-CMP processing and migration to minimize event-coupling artefacts. These artefacts represent cross-talk energy between various wave modes and include the unwanted part of the wavefield. We show how to produce detailed subsurface images within the region of interest (exploration prospect only) by applying the closely tied processes of prestack event enhancement and separation, well-driven time processing for velocity model building, and final event-based prestack depth imaging. Results show enhanced structural detail and good continuity of principal volcanic units and deeper reflections, suggesting a faulted $0.6-0.9 \mathrm{~km}$ thick layer of Cretaceous sediments in the proximity of well 209/09-1. Our interpretation complements existing low-resolution geophysical models inferred from gravity and wide-angle seismic data alone.
\end{abstract}

Keywords: Sub-basalt imaging, North of Shetland, Erlend Volcano, generalized discrete Radon transform, local moveout attributes, mode conversions

\section{Introduction}

The aim of this case study is to examine the feasibility of using both PP and converted waves for imaging high-velocity layers (HVLs) and sub-HVL reflectors (after Purnell, 1992). HVLs that overlay lower velocity structures hinder exploration in potential hydrocarbon producing areas of the North of Shetland, UK (Fig. 1). These layers are typically associated with the Cretaceous and Tertiary igneous rocks of the British Igneous Province representing igneous centres, extensive sill complexes, and widespread flood basalts (Gatliff et al., 1984; Stoker et al., 1993; Jolley \& Bell, 2002). Seismic-velocity data analysis in the North Atlantic Margin is well documented (Planke et al., 1999; Ogilvie et al., 2001; Fruehn et al., 2001). It appears that
P-wave propagation is hindered by post-critical phenomena typical for high-velocity structures. Moreover, the weak subbasalt PP reflections interfere with strong (surface-related and internal) multiples and converted-wave arrivals caused by dramatic elastic-impedance contrasts between the HVLs and surrounding relatively soft sediments. The precise mechanism for such noise varies from case to case (Martini \& Bean, 2002; Maresh \& White, 2005). Preliminary multi-wave-type or eventbased imaging, illumination and forward modelling studies (Purnell, 1992; Barzaghi et al., 2002; Van der Baan et al., 2003) have been particularly encouraging, suggesting the possibility of imaging beneath HVLs using converted waves generated at the interface between formations with strong velocity contrast.

\# Paper presented at the 67th EAGE Conference \& Exhibition, Madrid, Spain, 13 - 16 June 2005 


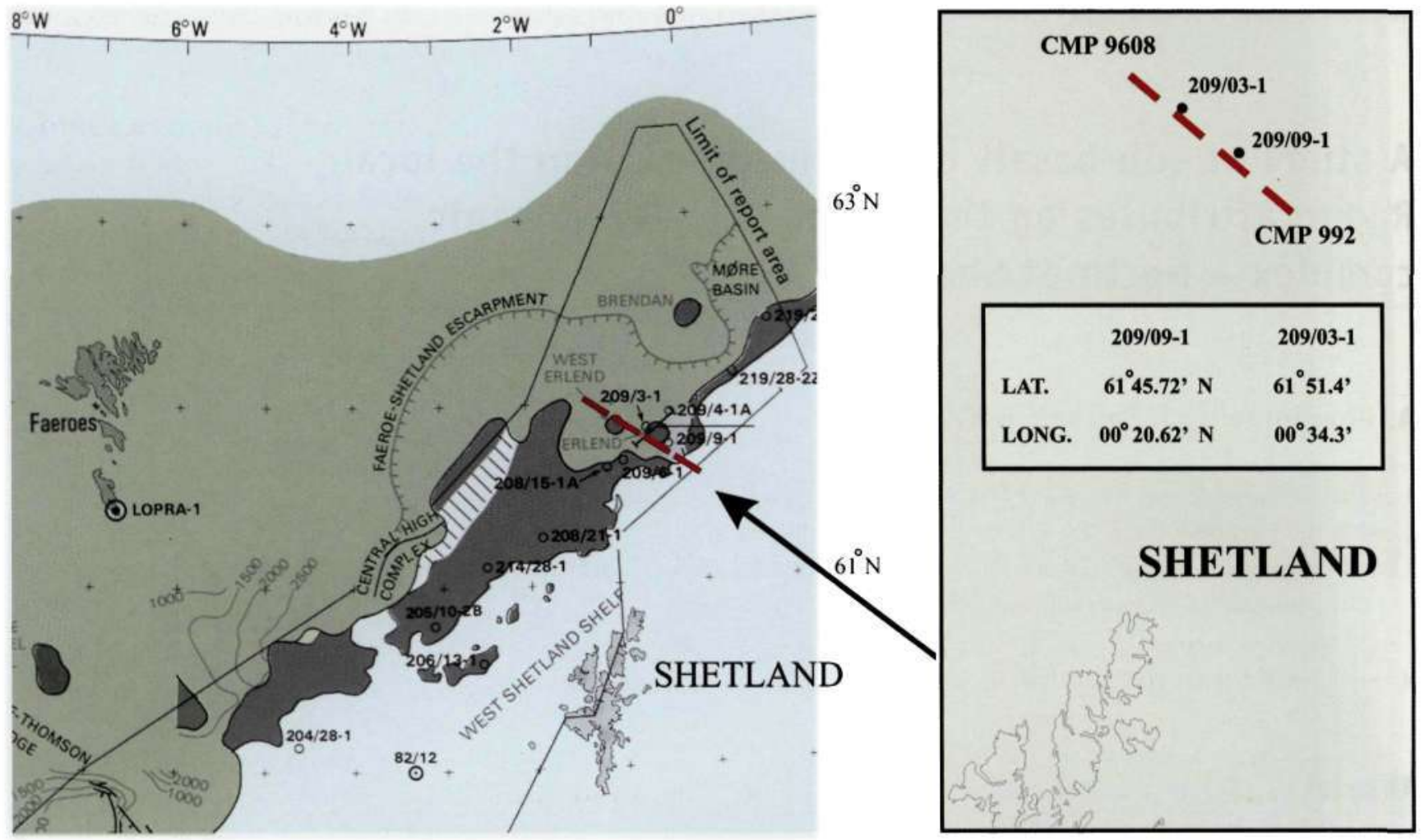

Fig. 1. Study area (UK sector of the North Sea, quadrants 208 and 209, the Faeroe-Shetland Escarpment): geological map (courtesy of BGS/NERC), well locations and schematic CDP locations for the profile (CDP 992-9608); enlarged schematic illustration of the survey geometry (well locations along the line are indicated).

Traditionally, wide-angle data have been used to derive a starting large-scale velocity model by traveltime inversion (Fruehn et al., 2001). Marine streamer data at moderate offsets of less than $6 \mathrm{~km}$ can be reprocessed using advanced noise attenuation techniques specifically designed to reduce uncertainties of short-wavelength velocity perturbations (0gilvie et al., 2001). In this case, non-PP or mode conversions often cause degradation of seismic sections and should be eliminated. On the other hand, a straightforward single-step prestack time migration (PSTM) or prestack depth migration (PSDM) combining all modes (events) is usually not reliable because events migrated with inadequate combinations of velocities produce severe migration artefacts due to frequency dependent event coupling (Takahashi, 1995). Since distortion is expected to be limited for data characterized by a single event, a domain transform or wave-equation complete wavefield separation is usually required to minimize cross-talk energy between individual events (Dillon et al., 1988). In practice, interference between various events on prestack gathers always prevents the desired clear separation of events (Martini and Bean, 2002; Spitzer et al., 2003).

To facilitate event-oriented data pre-conditioning and to accomplish event selection during the migration process, we applied the decoupled prestack migration formula to both vector and pressure recordings (Droujinine, 2003, 2005). According to the elastic migration principle (Kuo \& Dai, 1984), we migrated data using a combination of velocities and event enhancement amplitude weights suitable for each event. This makes it possible to avoid misprocessing and misinterpreting non-PP arrivals as primary PP reflections, without applying labourintensive pre-processing that involves the use of handpicked mutes or interactive interpretations based on calculations of the traveltime response (Barzaghi et al., 2002; Van der Baan et al., 2003). In event-based migration with accurate velocities, migrated energy from assumed wave-propagation modes coincides with the actual reflector, although different parts of this reflector are imaged with each event (Kuo \& Dai, 1984).

In this paper, we apply the event-based decoupled imaging approach to the $2 \mathrm{D}$ marine streamer dataset (courtesy of Norsk Hydro) acquired with standard towed streamer in the study area (see Table 1). The P-velocity model is constrained by good quality sonic logs and check shots. Pre-processed data have typical problems in basalt-covered regions: poor PP signal and strong coherent noise represented by remnants of multiples, refractions and converted waves (see Table 2). The water depth in this area is less than $0.4 \mathrm{~km}$, and the basalt is some $1.2 \mathrm{~km}$ below the sea surface. Referring to previous modelling experiments (Purnell, 1992), it is envisaged that $3 \mathrm{~km}$ offsets may be sufficient to observe non-PP reflections.

Initially, we applied conventional PP processing of the data set, including wave-equation PSDM. Shot gathers were depth migrated using the interval velocities available from isotropic 
Table 1. Acquisition parameters of the seismic survey.

\begin{tabular}{ll}
\hline Acquisition & Towed streamer \\
Area & North of Shetland \\
Company & Norsk Hydro \\
Year & 1994 \\
Source & Volume Airgun \\
Number of wells & 2 \\
Number of shots & 2155 \\
Number of receivers & 240 \\
Min offset (m) & 95 \\
Max offset (m) & 3083 \\
Shot spacing (m) & 25 \\
Receiver spacing (m) & 12.5 \\
Record length (sec) & 3.5 \\
\hline
\end{tabular}

Table 2. Key to the notation used in many of the figures.

\begin{tabular}{ll}
\hline Symbol & Definition \\
\hline PP & Primary PP reflection. \\
WB & Water-bottom reflection. \\
P & PP reflection from the base of Pliocene. \\
0 & PP reflection from the base of Oligocene. \\
E0 & PP reflection from the top of Middle Eocene. \\
TB & PP reflection from the top of basalt (Top of Basic \\
Volcanics). \\
BB \\
PP reflection from the base of basalt (Base Tertiary \\
Unconformity) that can interfere with the asymmetric \\
P-to-S mode conversion PPSP at the TB interface. \\
G1 \\
Group of events containing PPSP (downgoing P wave and \\
upgoing S wave with conversion to P wave at the sea \\
floor) arrivals associated with high-contrast interfaces \\
and overprinted by surface-related or water-layer peg-leg \\
multiples. In a physical model experiment, Tatham et al. \\
(1983) observed the converted S-waves from P-waves at \\
the interfaces. PPSP events and multiples are separated \\
during event-based PSDM. \\
Group of events containing PSPPSP (symmetric local mode \\
conversion at the TB and BB interfaces during transmission, \\
S travelling inside basalts) arrivals overprinted by inter- \\
basalt and peg-leg multiples. Along the PSPPS path, a \\
wave converts from S to P upon exiting the basalt and \\
from P to S upon re-entering the basalt (Purnell, 1992). \\
PSPPSP events and multiples are separated during \\
event-based PSDM. \\
F,H1,H2 \\
First-order water-bottom (PPPP) multiple suppressed \\
during event-based PSDM. \\
multiples, diving waves, etc.) that arrive ahead of PP \\
reflections (including the water-wave cone) at larger \\
C2
\end{tabular}

Dix inversion of root-mean-square (RMS) velocities and subsequent time-to-depth mapping, as suggested earlier (Robein \& Hanitzsch, 2001). From inspection of preliminary results (not shown), we concluded that a major problem of building a velocity model for data such as these is the accurate mapping of the whole volcanic succession and the estimation of sediment velocities beneath the basalt structure. Since both multiples (water-bottom, peg-leg, inter-basalt, etc.) and converted waves have a profound impact on image quality, the choice of migration will depend upon the possibility of handling those arrivals that mask the primary signal. This emphasizes the need of event-based imaging and optimal velocity focusing strategy being chosen.

The paper is organized as follows. In section 2, the eventbased imaging technique is briefly outlined, and an entire processing sequence is discussed in detail. Rather than focusing on theoretical aspects, this section emphasizes the key implementation issues that noticeably improved image quality. Section 3 describes sub-basalt imaging results in the study area. Our seismic-well data analysis reveals the most significant geological and geophysical challenges that affected the quality of migrated data. In section 3, we present the adopted processing solutions while focusing on each step of the workflow: pre-CMP signal enhancement and wavefield separation, prestack time/depth migration and velocity updating. Crucial to this model-based approach is our multistep derivation of an accurate, anisotropic velocity model using measured local wavefront attributes and calibrated well data.

\section{Methodology}

Much of the methodology that follows was motivated by the work of Droujinine (2005), in which he derived and tested numerically the Generalized Discrete Radon Transform (GDRT) of wavefield separation, migration velocity analysis and prestack migration suitable for imaging beneath HVLs. The GDRT is a discrete version of the Generalized Radon Transform (GRT) (Beylkin, 1982) that extends conventional linear or parabolic slant-stack data transformations used in seismic processing (Yilmaz, 2001). To obtain a good seismic image along the 2D profile, we have applied the GDRT PSDM workflow (Droujinine, 2003) aimed at effectively removing strong superfluous events (coherent noise) while enhancing weak valuable events, especially in the low frequency components of the data. Table 2 contains a list of principal events (both signal and noise) to be enhanced, migrated or attenuated. Appendix explains how slant stack parameters, kinematic wavefront attributes or corresponding local moveout operators can be directly estimated from seismic data without recourse to an initial velocity model. 


\subsection{Wavefield separation}

Primaries and multiples can be separated in the parabolic Radon domain because of their different RMS velocities (Spitzer et al., 2003). Similarly, event separation can be achieved by means of local moveout operators (see Appendix), used to increase the PP-wave signal-to-noise ratio and to identify nonPP events not interpretable on the PP sections. The GDRT filter is applied to common-midpoint (CMP), common-source (CS) or common-receiver (CR) gathers. Assuming the offset domain, the program computes a model of primary and multiple events by taking into account local traveltime approximations in Appendix. The GDRT algorithm finds a representation in the model domain that, in a least-squares sense, describes the input data best. The justification for event modelling is that it allows separation of principal arrivals described in Table 2. GDRT represents data decomposition into user-defined parabolic (beam-stack) segments (A1) using the least squares method in the frequency-space domain (F-X) for each frequency of the band pass defined by $f_{\min }$ and $f_{\max }$. According to the elastic Huygens' principle (Kuo \& Dai, 1984), GDRT simulates the input data as a linear combination (superposition) of the individual wave modes (PP, converted waves, refractions, etc.). The data are split into several frequency bands, the detection occurs on the higher bands, and a selective attenuation is made so as to preserve the lower bands, which contain more primary energy. The GDRT decomposition consists in carrying out semblance scans (stacks) according to the various values of the $(P-Q)$ attributes in eq. (A1), then a spatial deconvolution of the obtained spectrum. The scan of parabolas is defined by the position $\left(\tau, X_{0}\right)$ and an increment between parabolas $(\Delta \tau$, $\left.\Delta X_{0}\right)$. The user also specifies the $(P-Q)$ attribute lower-upper limits and increments $\left(P_{\min }, P_{\max }\right),\left(Q_{\min }, Q_{\max }\right)$ and $(\Delta P, \Delta Q)$. The attribute range and increment values are chosen so that it is possible to separate events of interest and to eliminate alias contributions while still including undistorted events passed by a given window function. The events are defined by the (pass or reject) sub-range or threshold values $\left(P_{\min }^{0}>P_{\min }\right.$, $\left.P_{\max }^{0}<P_{\max }\right),\left(Q_{\min }^{0}>Q_{\min }, Q_{\max }^{0}<Q_{\max }\right)$ or $\left(P_{0}, Q_{0}\right)$. For example, events corresponding to parabolas with the $Q$-attribute greater than the threshold $Q_{0}$ can be considered as multiples, whilst events corresponding to parabolas smaller than this threshold can be deemed to be primary events. The difference between data and the sum of modelled events is interpreted as residual noise. In practice, the multiple-only gather often contains some residual primary energy and vice versa (Yilmaz, 2001). GDRT subtracts from the input gather the model of events or the individual event plus the residual noise. During event identification and velocity analysis sessions, GDRT enables users to view the (P-Q) attribute (two-parameter) semblance panels of Droujinine (2005) as conventional (single-parameter) effective velocity spectra. GDRT offers the following benefits: (1) analysis may be carried out on an existing grid of CMP locations as commonly defined for RMS velocity scans, (2) it may speed laborious event picking on large projects without decreasing event resolution, and (3) it eases quality control of the global reflection moveout (Yilmaz, 2001) or its anisotropic counterpart (Thomsen, 1999) within a user specified offset range.

\section{Remark 1}

In an ideal GDRT decomposition events are well separated in the $(\tau-\mathrm{P}-\mathrm{Q})$ domain. A region in the $(\tau-\mathrm{P}-\mathrm{Q})$ domain that represents events of interest (e.g. primaries) can be isolated. If the primaries are muted, the remaining part of this domain contains only multiples that can be subtracted from the input data.

\section{Remark 2}

The particular case of (global) parabolic event filtering can be considered within the framework of GDRT if we set $h_{0}=X_{\min }$, $\Delta \mathrm{h}=\mathrm{X}_{\max }-\mathrm{X}_{\min }$ and $\mathrm{P}_{\min }=\mathrm{P}_{\max }=0$ (an example of global parabolic events is residual moveout in the migrated domain). Whereas in the parabolic Radon domain the contributions of traces at different offsets are stacked over globally parabolic events, the same can be done along globally linear dipping events (conventional slant stack or $\tau-p$ transform) by setting $\mathrm{Q}_{\min }=\mathrm{Q}_{\max }=0$.

\subsection{Velocity model building}

The velocity model building for prestack migration in the area has two equally important tasks - the preliminary interpretation of the complicated HVLs and the sediment velocity changes around and beneath these units. Following Robein and Hanitzsch (2001), RMS velocity analysis is initially applied to the CMP data based on the velocity approximation $V(x, \xi)=$ $V_{0}(\xi)+\Delta V(x, \xi)$. Here, $V_{0}(\xi)$ is the one-dimensional guide function derived from the sonic logs and constrained by the regional regression formula (0gilvie et al., 2001); the velocity perturbation $\Delta V(x, \xi)$ is a given function of lateral and vertical coordinates $x$ and $\xi=t$ (time) or $\xi=z$ (depth). In order to estimate this velocity perturbation, two locked window displays are used. The first window shows the moveout-corrected CMP gather on the vertical time axis (TO in ms) and the second one shows the semblance RMS velocity panel. Semblance velocity scans are generated at given CMP locations (e.g. every $500 \mathrm{~m}$ ), with the central velocity function corresponding to the velocity $\mathrm{V}_{0}$. At locations where the moveout-corrected CMP gathers do not demonstrate optimal flattening, a residual moveout correction can be picked and applied. These changes are shown on the semblance display. The gathers and semblance displays can also be used to check that the model contains a sufficient number of surfaces to define the velocity variation 
of the data properly. If the gathers show good flattening at the top and base of a layer but not at intermediate points, this indicates that the layer should be sub-divided so that different velocities can be applied.

If the model contains steep dips and/or strong lateral velocity contrasts, then the velocity $\Delta \mathrm{V}(\mathrm{x}, \xi)$ may no longer be estimated correctly. In these cases, structural updating is necessary. For most cases, this can be achieved adequately by using the latest model for migration of an unmigrated time stack volume, upon which a structural re-interpretation can be performed. In cases of extreme complexity, as for example in this study imaging below HVLs, the RMS velocity analysis may not give sufficient confidence of the optimal focusing and correct structural positioning. It is then necessary to use prestack time and depth migration runs for a series of shot or CMP gathers, which can then be structurally re-interpreted and interpolated to update the reference model.

The key step of the time-domain reference velocity model building is to apply the conventional Dix inversion to the stacking velocity field. Due to well-known uncertainties of Dix inversion (Lambaré et al., 2007), we focus our attention on the calibration between wells and seismic sections. Firstly, we build an initial isotropic velocity model, using existing well control and time horizon maps consistent with the available lithology columns. Secondly, we estimate the effective Thomsen's (1986) parameter $\delta$ from the mismatches between effective $\mathrm{P}$-wave velocities derived from surface seismic data after signal enhancement and compressional sonic logs after upscaling. Since the lack of structure permitted the assumption of a vertical axis of symmetry (VTI), we incorporate the following velocity matching formula (Hawkins et al., 2001)

$$
V \text { (flat dip) }=V_{0} \sqrt{1+2 \delta}
$$

where $V$ is the effective, normal moveout (NM0) or image-ray velocity and $V_{0}$ is the vertical velocity predicted by calibrated sonic logs or check shots. Although the meaning of $\delta$ is less obvious than that of other anisotropy parameters, it has a clear formal definition, relating to the second derivative of the phase velocity function at normal incidence to the vertical P-wave velocity (Thomsen, 1986, 1999). Since the first derivative of phase velocity at normal incidence is zero, $\delta$ is responsible for the angular dependence of the phase velocity in the vicinity of the vertical axis.

Evidently, confidence limits $\pm \Delta \delta$ of the parameter $\delta$ depend on the quality of the seismic-well tie and have nothing to do with the horizontal velocity. Under the short spread assumption $(X / z<1)$ and weak lateral velocity variations $\left(|\Delta \mathrm{V}(\mathrm{x}, \xi)| / \mathrm{V}_{0}(\xi)\right.$ $\ll 1)$, the VTI moveout is still hyperbolic and is controlled by only the parameter $\delta$ (Thomsen, 1986). Hence, the initial values of $\delta$ can be checked by running 'sensitivity tests' with moveoutcorrected CMP gathers for inspection of their flatness. All these tests are evaluated for the given subset of CMP gathers and a revised anisotropy parameter grid is calculated over the target region. This grid can then be subjected to edits and smoothed as for the initial model loading.

\subsection{Prestack migration and velocity updating}

Depth model building is usually an iterative process that starts from picking the key horizons on a time migrated section obtained with a migration velocity $V_{\text {mig }}$. The better the image of the time migrated section, the better the depthdomain velocity model. When there is no strong heterogeneity and high dips, reflectors are imaged at the zero-offset time $t_{0}$ and $V_{\text {mig }}$ is close to the stacking velocity $V_{\text {stack }}\left(t_{0}\right)$. Once $V_{\text {stack }}$ has been determined during pre-processing, the pre-processed subsets are migrated several times, using conventional Kirchhoff PSTM (e.g. Robein \& Hanitzsch, 2001) for major wave types identified during pre-processing, as outlined by Barzaghi et al. (2002). We begin with a smooth 2D model for which we search the principal reflections after PSTM has been applied with the velocity $V_{\text {mig }}=V_{\text {stack }}$. This leads in general to non-flat image gathers and requires additional (e.g. one or two) passes of PSTM with updated $V_{\text {mig }}$ until we reach the optimum flatness. Since the imaging is kept in the time domain, relative insensitivity to errors in $V_{\text {mig }}$ results in a moderate loss of focusing.

In contrast to PSTM, PSDM is a more accurate migration that is much more sensitive to the choice of migration velocity. Therefore, it needs many iterations of migration velocity updating and migration before proceeding through final interpretations (Jones, 2003). At least three iterations are necessary to obtain a reliable PSDM velocity model:

1. Of particular importance is the need to incorporate the key time horizons (output of PSTM) into the reference interval velocity model that accounts for all available sources of velocity and geological information. At this stage, time stacking velocities are converted to interval velocities and then to depth interval velocity (Yilmaz, 2001). With horizon intervals and depth interval velocities a velocity grid $V^{(0)}$ is constructed and used for the initial PSDM (iteration 1).

2. The output of iteration 1 helps the interpreters to identify the HVLs in the depth domain in order to reduce uncertainties of the time-domain interpretation (Lambaré et al., 2007). It is then possible to generate the final depth-domain top basalt and base basalt interfaces and to create the modified velocity field $V^{(1)}$. Here, we make use of the velocity $V^{(1)}$ to compute depth migrated post-stack volume and depth migrated prestack volume (iteration 2) to facilitate velocity updating beneath base basalt. Note that although detailed interpretation of volcanic units is essential to capture the rugosity of basalts in the region (Planke et al., 1999; Ogilvie et al., 2001), the depthconverted horizons should be cleaned by filtering out cycle skips and filling the gaps by extrapolating from wellconformed portions of each horizon. 
3. The short-wavelength refinement of the velocity field $V^{(1)}$ is then carried out in the post-migrated domain through the analysis of the residual moveout (RMO) on the common image gathers by performing velocity scans in the areas of low signal-to-noise ratio, especially beneath the base basalt horizon. As part of the updating process, bending ray tracing occurs for the subsurface locations where the RMO corrections were picked (Jones, 2003). Since RMO is normally picked in the in-line direction, smoothing should be applied to the residual velocity model to minimize coarseness in the cross-line direction. The updated velocity model $V^{(2)}$ now can be used for final PSDM (iteration 3 ).

The high-quality GDRT depth migration procedure (Droujinine, 2003, 2005) implemented as the fast wavepath or Fresnel-aperture PSDM is utilized to provide depth images for model building as well as for the final output over the region of interest. This procedure is applied to map the reflections to their true vertical depth locations $(z, \xi)$ for both PP and converted P-to-S-to-P waves (see Table 2). As with conventional Kirchhoff migration, the two key migration steps are as follows: (1) calculation of multi-wave-type traveltimes between the image points $(z, \xi)$ and observation points; (2) using these traveltimes to map the reflections to the image points. Input data are pre-processed shot gathers after GDRT wavefield separation, followed by traveltime computations in terms of local wavefront attributes in Appendix.

\subsection{Concluding remarks}

Based on the GDRT theory, this section discussed the three basic steps of the processing stream: (1) data pre-processing, (2) reference model building, and (3) prestack migration and velocity updating. Here, we emphasize that the quality of PSDM is not only dependent on the migration algorithm, but also relies on the quality of data pre-processing, a priori information, and iterative velocity updating constrained by regional geology and well data. Using robust PSDM algorithm combined with event decoupling, and incorporating PSTM into model building, we can obtain high quality sub-basalt image.

\section{Results of sub-basalt imaging}

\subsection{Geological Background}

Following the recent geological analysis by Jolley and Bell (2002), the overall objective of our study is to consider evidence for the depositional environment of the strata of the Erlend Volcano (Fig. 1) revealed by seismic reflections. The existence of the Erlend Tertiary plutonic centre to the north of Shetland was recognized by the pronounced positive gravity and magnetic anomalies (Gatliff et al., 1984). This centre consists of a buried, partially-eroded shield volcano underlain by a basic pluton.
Unlike the related onshore central volcanic complexes, it retains a reasonably complete succession of volcanic lithologies (Stoker et al., 1993). Earlier gravity modeling and seismic mapping studies by Gatliff et al. (1984) have demonstrated that the Erlend volcano exhibits several distinct horizons that are radially disposed around a central vent. These horizons represent the original depositional attitude of the lava flows. Analysis of geophysical data has shown that there is a lava escarpment that is interpreted as a hyaloclastite deltaic sequence which formed as lavas entered the basin, chilled out and fragmented, producing a prograding sequence of bedding volcanic breccias. The basalts of late Palaeocene age (Stoker et al., 1993) provide strong reflections that can be traced across the Faeroe-Shetland Trough into the northern parts of quadrants 208 and 209 (Fig. 1). Several hydrocarbon exploration wells have penetrated thick sequences of subaerial facies basaltic lavas and subaqueous volcanic breccias, overlying Palaeogene and Cretaceous sedimentary rocks interbedded with medium to fine-grained silicic igneous rocks (Stoker et al., 1993; Jolley \& Bell, 2002).

\subsection{Seismic well data analysis}

We begin with the analysis of available input seismic and well data. A logical sequence consists of a systematic progression from well log conditioning, to seismic quality control (including spectral analysis) and finally preliminary identification of some events listed in Table 2 and initial velocity estimates with particular attention paid to the recent regional studies of Planke et al. (1999) and Ogilvie et al. (2001).

Interpreted wireline logs from wells 209/09-1 and 209/03-1, on the SE and NW flanks of the former volcano, proved total volcanic successions over $453 \mathrm{~m}$ (basic and acid volcanics) and $836 \mathrm{~m}$ (pyroclastic extrusive) in thickness, respectively (compare Figures $2 \mathrm{a}$ and $2 \mathrm{~b}$ ). Jolley and Bell (2002) provided a detailed analysis of the igneous lithologies encountered in these wells. Figure 2a confirms $150 \mathrm{~m}$, from $1172 \mathrm{~m}$ to $1322 \mathrm{~m}$ below rotary table (RT), of basic volcanics as well as $303 \mathrm{~m}$, from $1322 \mathrm{~m}$ to $1625 \mathrm{~m}$ below RT, of acid volcanic clastics, together with siltstones and sandstones. The check-shot timedepth pairs were used to 'tie' sonic logs to seismic.

The marine streamer dataset was acquired with standard towed streamer over offsets of traditional length. Details of 2D acquisition can be found in Table 1. The dataset was provided by Norsk Hydro after standard signal enhancement including source deconvolution and free-surface multiple attenuation. This is prior to our data pre-processing procedure. The data (multi-offset traces) acquired for a single shot is initially placed in a common-shot gather (Fig. 3). In Figure 3a (T-X display), one can identify and correlate the prominent reflections (events WB, P, 0, TB, and BB) as well as high-amplitude coherent noise (refractions $\mathrm{H}$ and multiples $\mathrm{M}$ ). The spectral characteristics of recorded common-shot data were examined through F-X power 


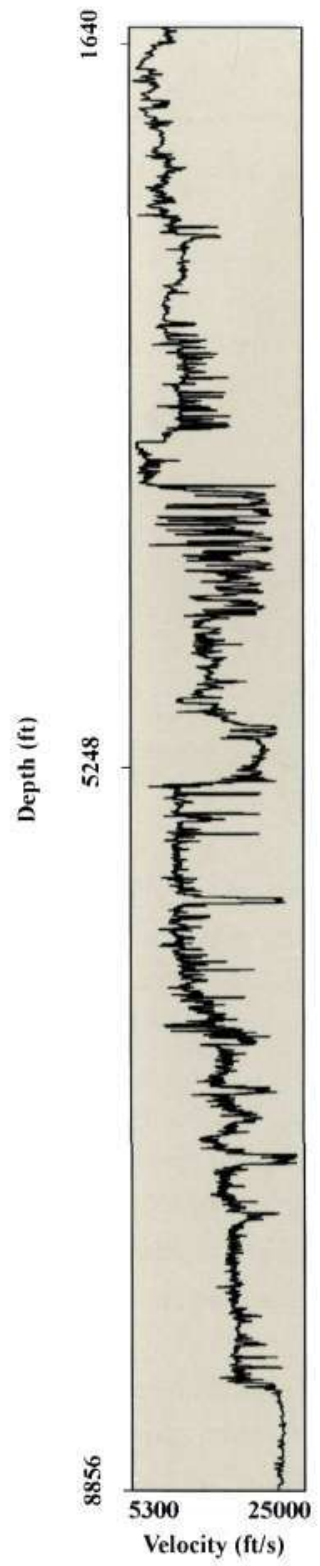

a.

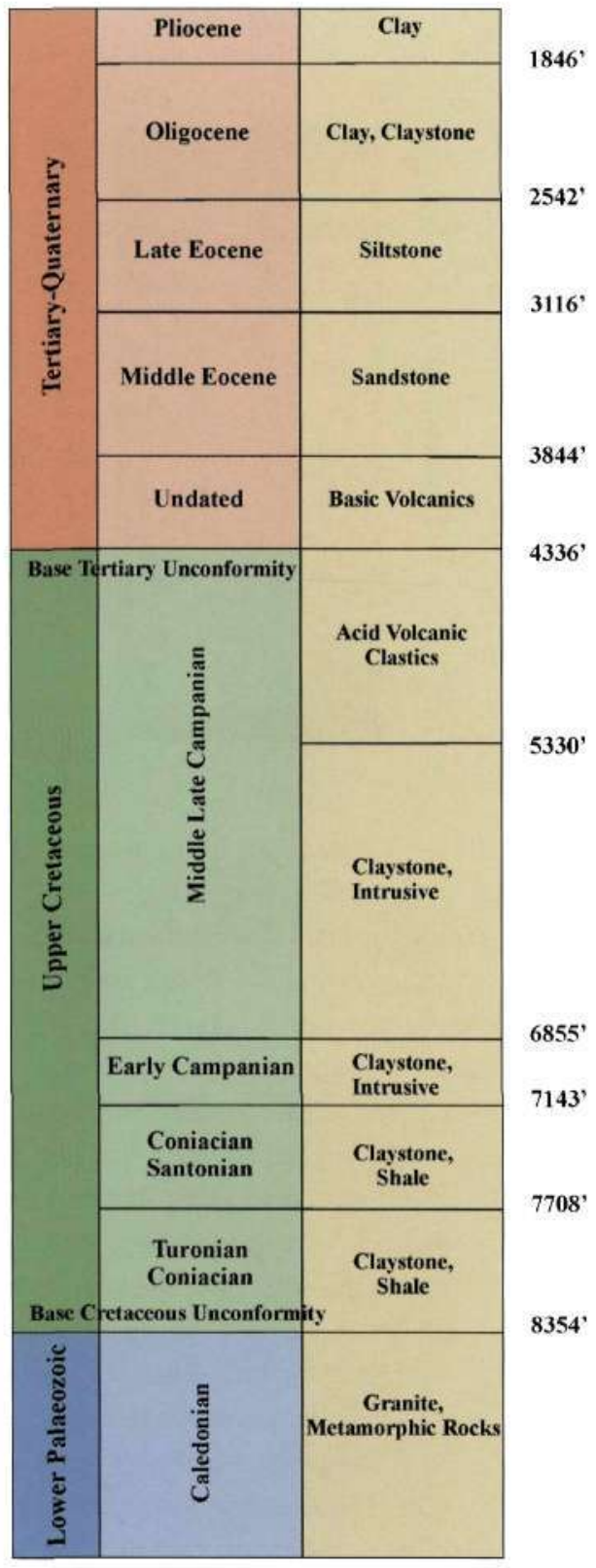

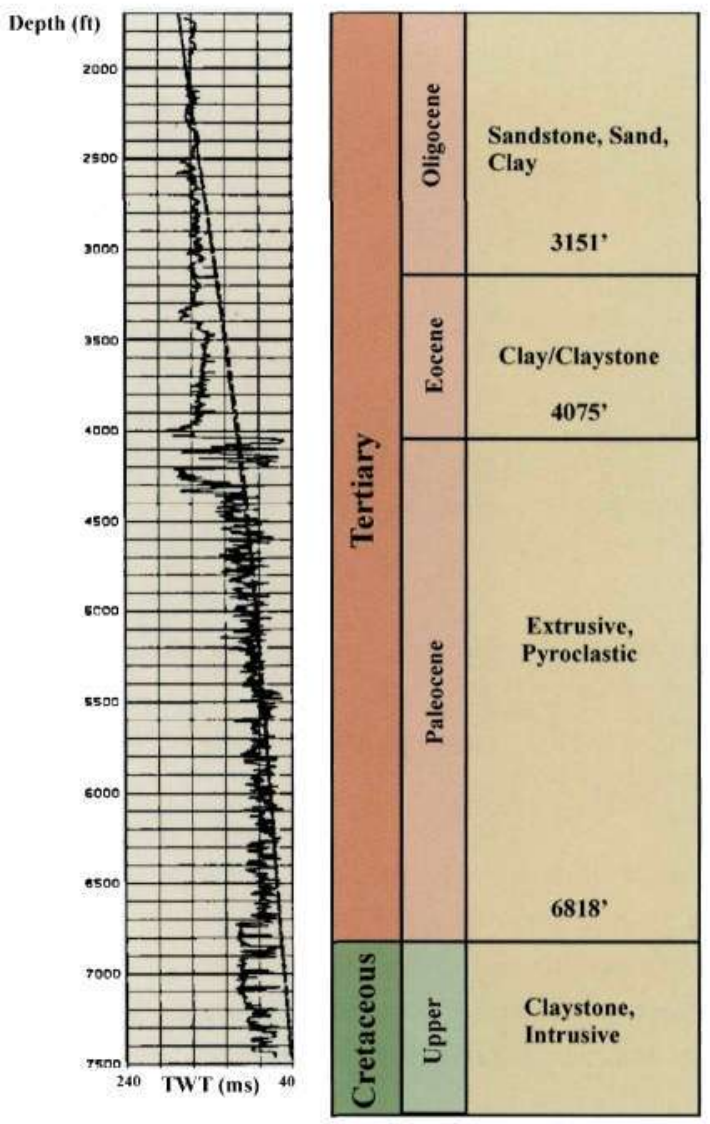

b.

Fig. 2. Calibrated $P$ sonic logs with the lithology and stratigraphy at the drill sites: wells (a) 209/09-1 and (b) 209/03-1 (courtesy of Fugro Robertson Ltd.). Shear sonic log data were not available. spectra (Fig. 3b) and average power spectra (Fig. 3c) for selected subsets of traces. The F-X spectrum in Figure $3 \mathrm{~b}$ shows the frequency dependent amplitude dimming at near and mid-range offsets. This is possibly due to some roughness of HVL boundaries at a scale similar to the seismic wavelength (Martini \& Bean, 2002; Maresh \& White, 2005). Attenuation of seismic energy within the HVLs caused by internal multiple scattering (Spitzer et al., 2003) is another mechanism that may explain the above amplitude dimming effect. The F-X spectrum in Fig. 3b also shows the increase of amplitudes at large offsets. According to Spitzer et al. (2003), this is mostly the contribution of converted-wave energy (P-to-S conversion at the TB boundary). In Fig. 3c, the spectrum contains little energy above $70 \mathrm{~Hz}$ and below $10 \mathrm{~Hz}$. The spectrum is flattened to within $5 \mathrm{~dB}$ from $15-50 \mathrm{~Hz}$. The focus of this paper is to address the poor data quality and acquisition limitations in order to apply a processing sequence that attenuates both coherent and random noise by preserving frequency content illustrated in Figs $3 \mathrm{~b}$ and $3 \mathrm{c}$.

\subsection{Prestack signal enhancement and wavefield separation}

The objective of prestack signal enhancement and wavefield separation is twofold: (1) signal enhancement (noise suppression and increasing the overall signal-to-noise ratio) and (2) GDRT separation of the key events listed in Table 2.

First of all, the recorded traces were converted to a zero phase band-limited signal applying a deterministic zero phasing. Zero phase signature processing was then carried out in order to remove the reverberation effects due to ghosts. Both filters were calculated based on the post-stack autocorrelation wavelet. 

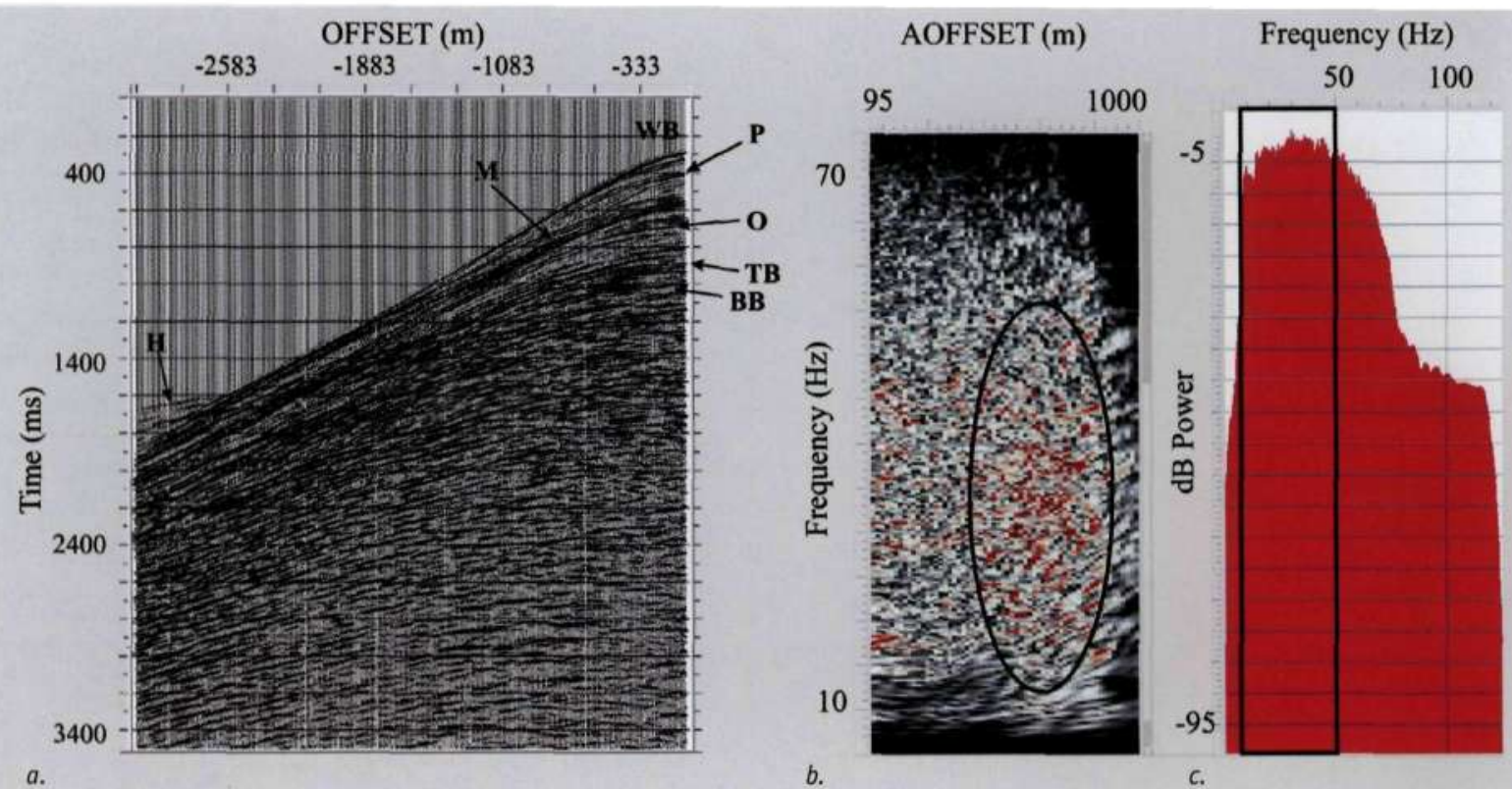

b.

c.

Fig. 3. Example single shot gather (CMP=992) before our data processing: $a$. T-X display (coordinate axes are two-way time and negative offset): b. F-X spectrum as a function of frequency and absolute offset (colour means energy power in dB: black colour corresponds to the minimum energy and red colour corresponds to the maximum energy); and c. Average power spectrum (in dB) computed directly from the autocorrelation of common-shot traces. Event notations are described in Table 2. The high-amplitude area is enclosed by the ellipse (middle panel). The rectangle indicated by solid lines fits the useful frequency bandwidth (right panel). The total signal bandwidth equals, or slightly exceeds, $100 \mathrm{~Hz}$.

It turned out that deconvolution had reduced the ringy character from the data and helped distinguish prominent reflections with ease, as shown in Fig. 3. However, the low-pass filter that was applied to enhance the low-frequency response and to remove some high-frequency random noise masked the resolution improvement. Initial multiple attenuation tests involved linear Radon and tau-p deconvolution. Generally, results were not successful because remnants of water-layer multiples (e.g. event M) can be seen in Fig. 3 .

The aim of subsequent GDRT filtering is to filter out the unwanted waveforms and to recover the desired signal that has been corrupted by additive noise, producing the corrupted signal. Shown in Fig. 4a is an example of prestack T-X domain shot gather prior to GDRT wavefield separation. It contains the source signals (waveforms), some of which we would like to retrieve (e.g. primary PP reflections in near-offset range and some non-PP arrivals in mid-offset range). The single pass of GDRT is implemented as follows (see Section 2.1 and Appendix):

1. Divide the input gather in Fig. $4 \mathrm{a}$ into small $(\tau-X)$ segments and apply the forward GDRT transform for every segment.

2. In the local $(r-P-Q)$ domain, we pick the most dominant $P$ and $Q$ values corresponding to strong unwanted events, and inverse transform these values into the $(\tau-X)$ domain. This yields the noise model segment containing refractions (events $\mathrm{H}_{1}$ and $\mathrm{H}_{2}$ ) as well as global (asymmetric) mode conversions $\mathrm{C} 1$, as indicated in Figure 4b.

3. Subtract the noise model from the input segment in the least-squares sense after trace matching (White, 1980).
The output gather in Fig. $4 c$ is obtained by repeating steps 1 - 3 for all the segments. Note that the GDRT preserves amplitudes and waveforms but can be severely aliased if the Nyquist condition of optimal spatial sampling is violated. Therefore, windows derived from semblance data in Fig. 4 limited to the $15-50 \mathrm{~Hz}$ pass band (Fig. $3 \mathrm{c}$ ) should be multiplied with the original GDRT to eliminate alias contributions while still including undistorted GDRT waveforms.

A comparison of Figs $4 \mathrm{a}$ and $4 \mathrm{c}$ shows that the process of GDRT filtering enhances spatial continuity of either PP or nonPP (C2) reflection energy while suppressing undesired events at near-or mid-offset ranges. Although cross-talk artefacts and remnants of more complicated paths are still observed in Fig. $4 c$, it is reasonable to assume that they will be negligible in the depth migrated domain. For the sake of comparison, Figure $4 \mathrm{~d}$ portrays the result of applying the Parabolic Radon Transform (PRT) algorithm to the same data traces (after Spitzer et al., 2003). As can be seen by comparing Fig. $4 \mathrm{c}$ and $4 \mathrm{~d}$, while both GDRT and PRT procedures have been effective in suppressing the strong refraction $\mathrm{H} 1$, it appears that PRT filtering enhances only arrivals $\mathrm{C} 2$ and refractions $\mathrm{H} 2$.

\subsection{Prestack time migration and initial velocity estimates}

The time processing process remains the important step in our sequence because the quality of the final depth image is strongly related to the quality of stacks or time migrated 


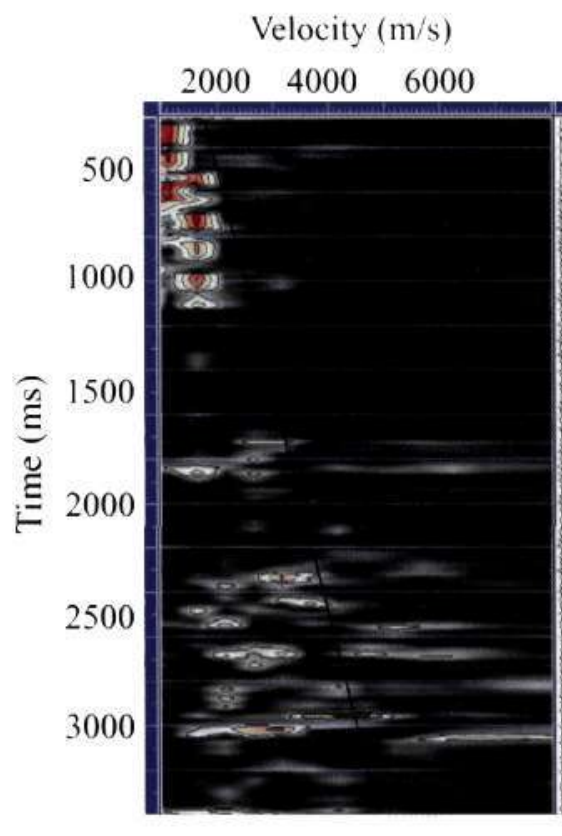

a.

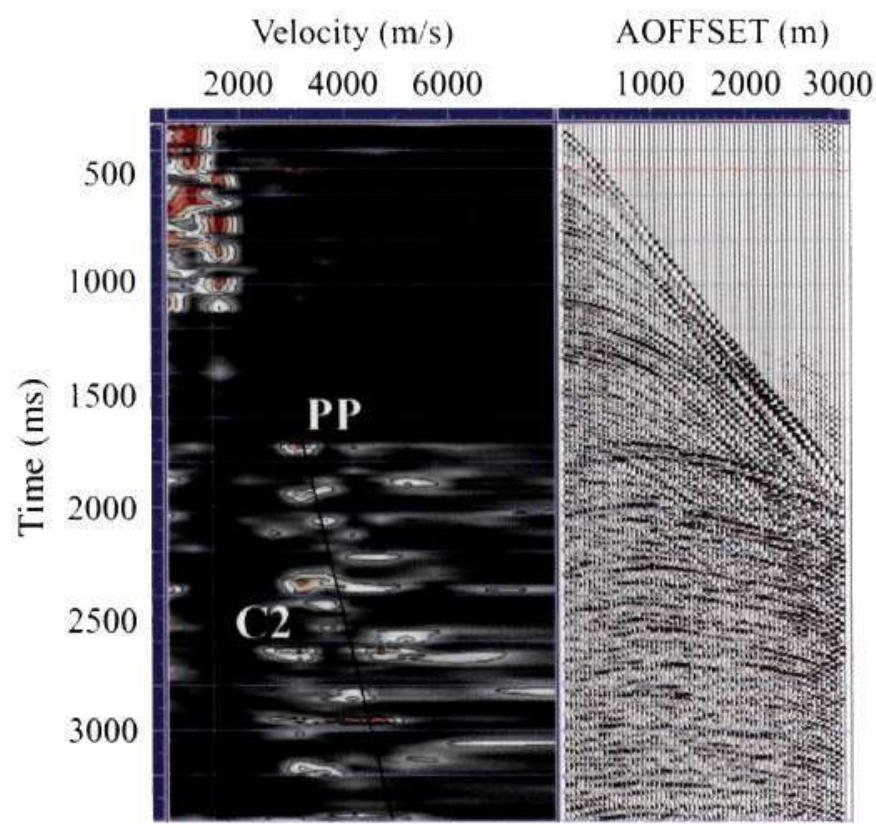

AOFFSET (m)

$10002000 \quad 3000$

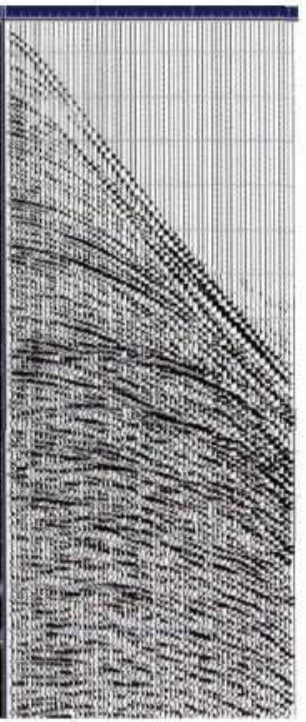

Velocity $(\mathrm{m} / \mathrm{s})$

$2000 \quad 4000 \quad 6000$

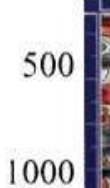

1500

2000

2500

3000
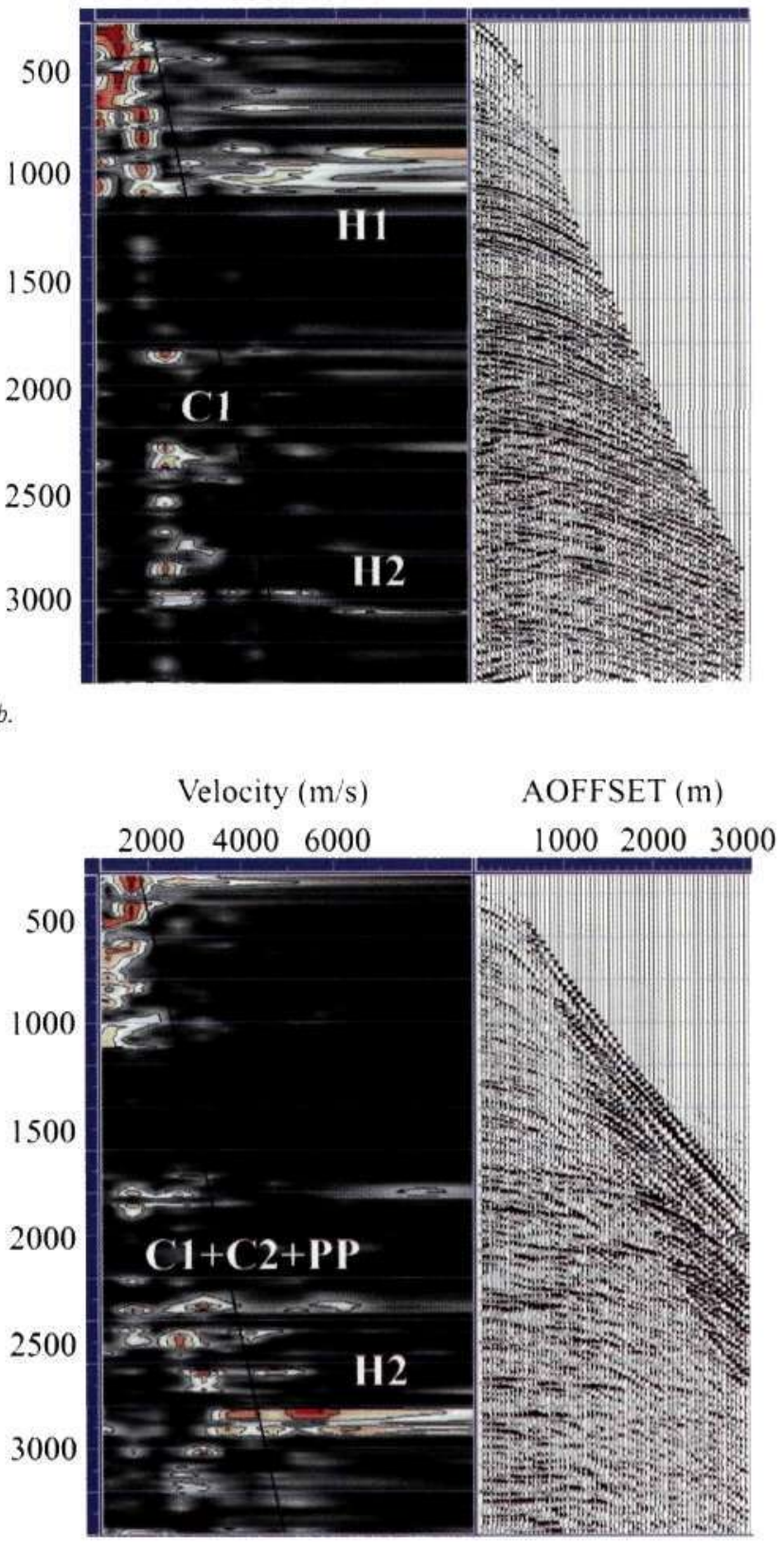

AOFFSET (m)

$1000 \quad 2000 \quad 3000$

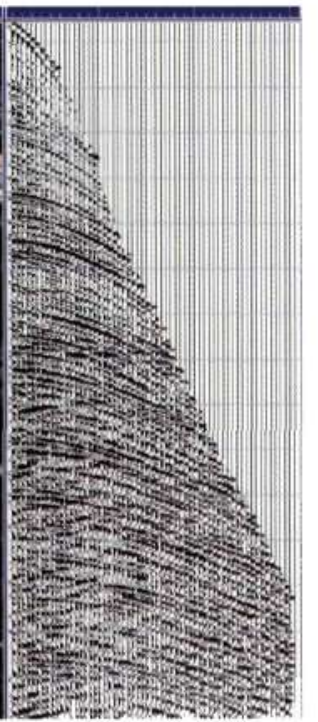

Fig. 4. Example velocity semblance spectra (left) derived from common-shot T-X trace panels (right) before and after signal enhancement and wavefield separation (CDP = 1792): $a$. Input data; $b$. GDRT prediction of coherent noise below the TB horizon; $c$. GDRT prediction of PP reflections below the TB horizon; and d. Sub-basalt PP signal enhancement using the parabolic Radon transform, as proposed by Spitzer et al. (2003). We focused our attention on the strong and isolated $\mathrm{C}_{1}, \mathrm{C} 2$ and PP events. Event notations are described in Table 2.

sections (Yilmaz, 2001; Robein \& Hanitzsch, 2001). In the present study, this process is tailored to build the reference model beyond the layered Earth assumption and to carry out prestack migration velocity analysis in the time domain (see Sections 2.2 and 2.3). Velocity model building for time migration started from estimating the reference velocity guide function $V_{0}(t)$. A comparison of $1 \mathrm{D}$ velocity-depth curves derived from sonic logs in Fig. 2 demonstrates that the linear gradient model assumption is a reliable approximation of this function similar to the existing regional velocity regression formula of 0gilvie et al. (2001). Next, points (picks) selected from the velocity semblance panels of pre-processed CMP data, optimally by comparison with QC NMO stacks, were used to generate a time velocity function $V_{\text {stack }}\left(x, t_{0}\right)$ for stacking and Dix inversion. The Dix inversion formula then transformed the stacking velocity into the reference 'sediment only' velocity perturbation 
$\Delta V(x, t)$. To reduce uncertainties of the reference isotropic model (Hawkins et al., 2001; Jones, 2003; Lambaré et al., 2007), we estimated the effective parameter $\delta$ using eq. (1). Confidence limits ('Top/Bottom Delta') of the parameter $\delta$ depend on the quality of the seismic-well tie. In noticing rather large uncertainties of the delta parameter beneath the base of basalt, one would suspect that inaccurate sub-basalt velocity model might have caused the initial poor image of Cretaceous sediments in prestack migration. In this study, non-hyperbolic anisotropic corrections (Thomsen, 1999) did not result in a higher image quality because of the $3 \mathrm{~km}$ offset limitation. To expedite the first multi-layered model, we analysed stacked NMO sections for preliminary interpretation while the reference sediment velocity model building was underway.

The prestack CMP data before and after GDRT were migrated using PSTM with the estimated migration velocity $V_{\text {mig }}\left(x, t_{0}\right)=$ $V_{\text {mig }}^{0}\left(x, t_{0}\right)+\Delta V_{\text {mig }}\left(x, t_{0}\right)$, where $V_{\text {mig }}^{0}\left(x, t_{0}\right)$ is the reference migration velocity derived from the stacking velocity $V_{\text {stack }}\left(x, t_{0}\right)$; residual curvature on the resulting time-migrated image gathers was used to obtain the velocity update $\Delta V_{\text {mig }}\left(x, t_{0}\right)$. The resolution of the estimated velocity models in Figs $5 \mathrm{a}$ and $5 \mathrm{~b}$ is low due to smoothing and editing of residual slowness in the velocity update. Aside from this, there is some indication of layering in Fig. 5b. It appears that the resulting velocity model in Fig. 5b exhibits the regional trend of velocities in the area. This model is more consistent with the interpreted HVL boundaries than that in Fig. $5 \mathrm{a}$, suggesting that migration velocity analysis of moveout-corrected CMP data after GDRT accomplished the process of adjustment of velocity model to structural interpretation. As with RMS velocities, the migration velocities of HVLs in Fig. 5 are generally low compared with volcanic interval velocities obtained from the well data in Fig. 2.

Figures $6 \mathrm{a}$ and $6 \mathrm{~b}$ show a comparison of time migrated CMP data before and after GDRT using the velocity models in Figs $5 \mathrm{a}$ and $5 \mathrm{~b}$, respectively. As can be seen, the result in Fig. $6 \mathrm{~b}$ demonstrates the improved image of overburden (e.g. events 0 and EO) and HVL boundaries (TB and BB) when compared with the result in Fig. 6a. Notice the significant reduction of highamplitude coherent noise around the two-way time (TWT) of $1700 \mathrm{~ms}$ in Fig. 6b (e.g. arrivals C1 and C2 in Figs 4b and 4c), outlined by the solid oval and the dashed box. Analysis suggests well defined HVL boundaries (except the BB geometry in the CDP range 2432 - 3872) and sufficiently strong intra-basalt reflection coherencies between the TB and $\mathrm{BB}$ horizons, acting as efficient PP reflectors and P-to-S energy converters. Despite the residual coherent noise including the seabed PPPP multiple (event M at $560 \mathrm{~ms}$ ), mode conversions and inter-basalt multiples, an improved time-domain velocity model can be constructed using the image of HVLs in Fig. $6 \mathrm{~b}$. Although iterative noise attenuation is quite challenging due to sparse source/receiver sampling and offset limitation, it is feasible to identify HVLs acting as efficient energy converters, whereas the interpretation of events outside the prospect region ( $C D P>4833$ ) remains highly ambiguous (not shown). Nevertheless, since the sub-basalt primary PP reflections are masked by migration artefacts and residual noise on the timemigrated unfiltered and filtered data (cf. Figs $6 \mathrm{a}$ and $6 \mathrm{~b}$ ), there is still not enough confidence that a significant subbasalt clastic section exists.
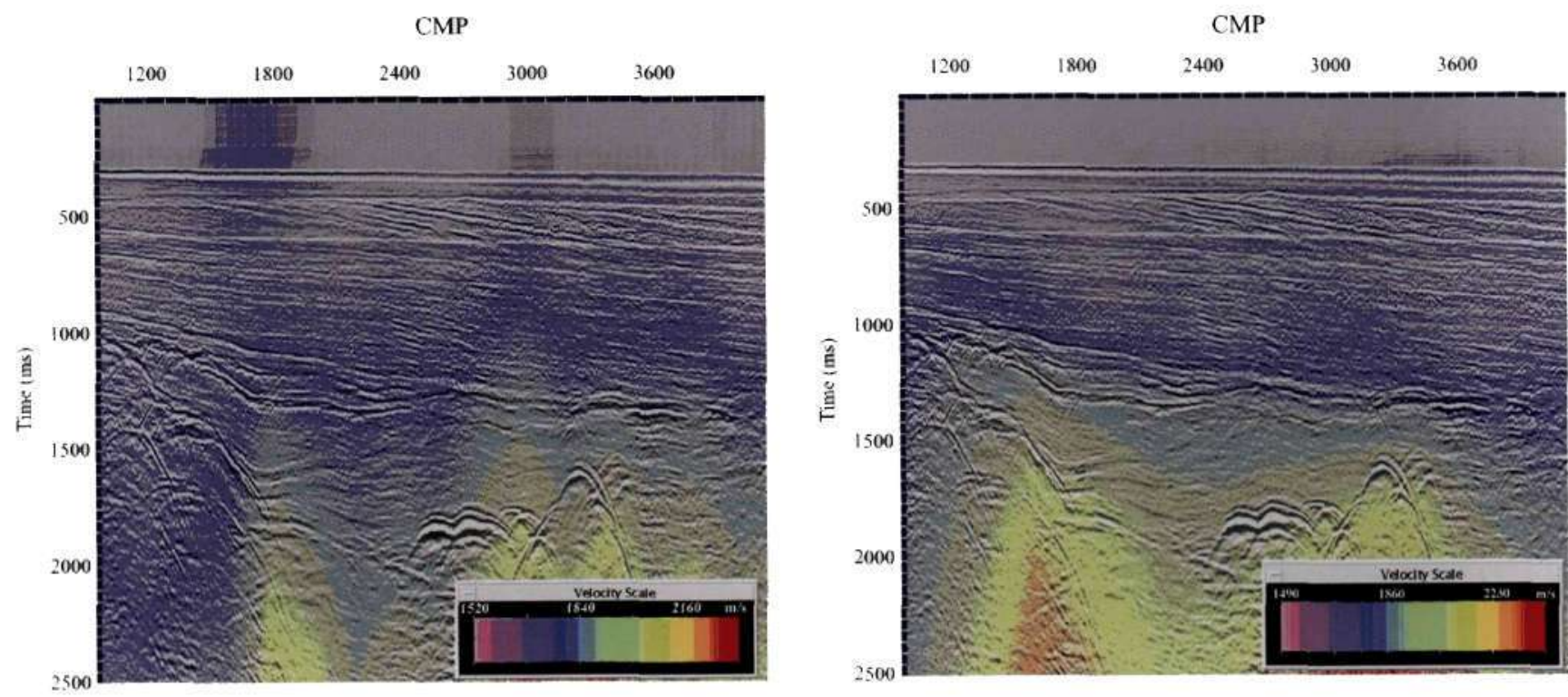

b.

Fig. 5. Results of picking maximum energy from effective velocity scan panels: velocity grids after smoothing and cubic interpolation (in m/s, no actual velocity-contrast interfaces). Input data are prestack CMP gathers (a) before and (b) after multi-pass GDRT signal enhancement. The velocity model is overlain on the stacked unmigrated section within the CMP range of interest. Grid points were distributed every $12.5 \mathrm{~m}$ in the $2 \mathrm{D}$ volume. The colour scale enhances maximum and minimum velocities. 

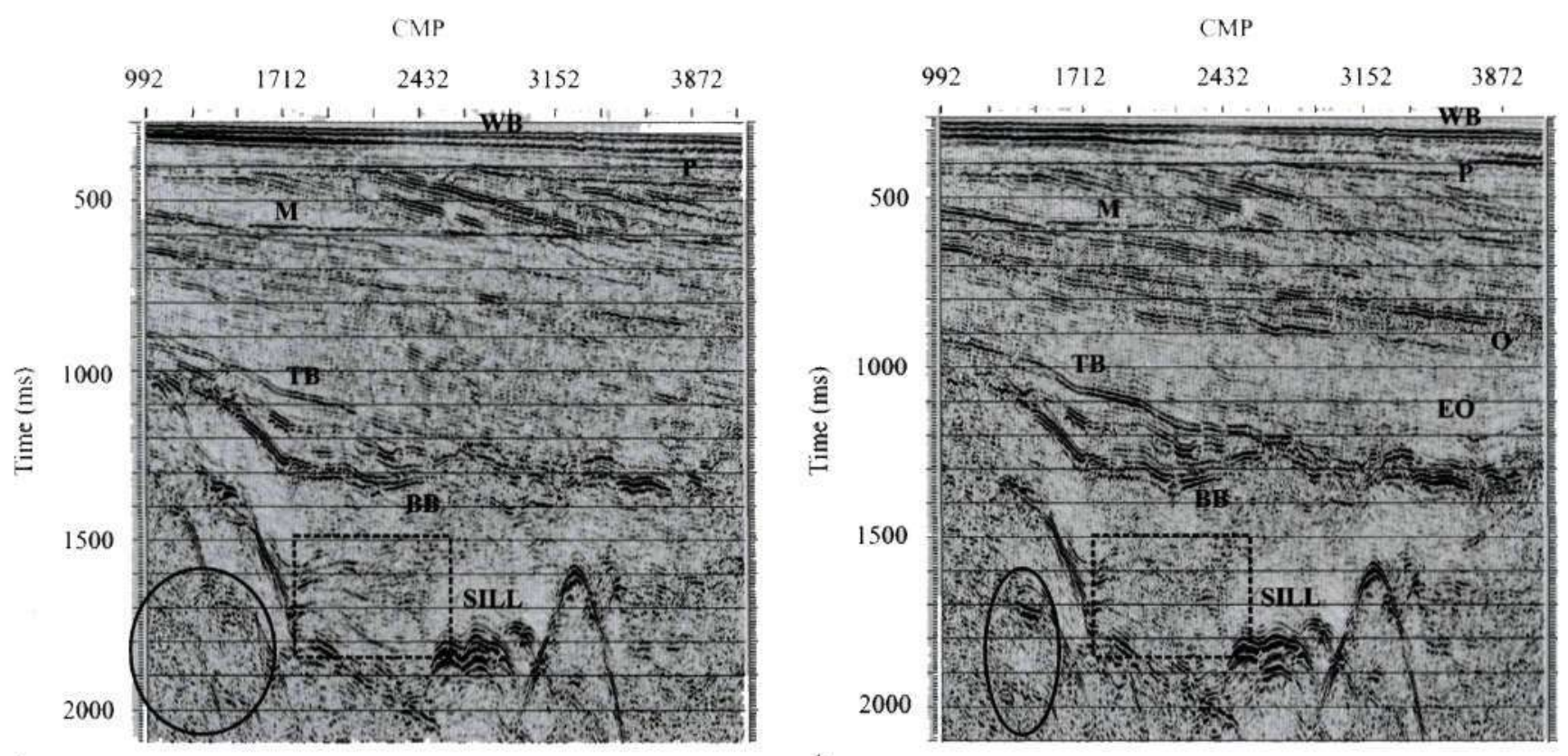

a.

b.

Fig. 6. Results of time processing of prestack CMP data (a) before and (b) after iterative GDRT signal enhancement using velocities in Figs $5 a$ and $5 b$, respectively. Identical trace scaling (no automatic gain control) was applied to both prestack time-migrated sections. Solid oval and dashed box indicate groups of coherent events that are discussed in the text. These groups contain events $C 1$ and $C 2$ seen in Figs $4 b$ and $4 c$, respectively. Event notations are described in Table 2.

\subsection{Prestack depth migration and final velocity updating}

Deficiencies of time processing outlined in Section 3.4 utter the need of depth migration (see Section 2.3) that can accommodate layers with vastly different elastic properties. The practical difficulty is that PSDM requires an accurate velocity model in depth and is a more labor-intensive operation. We built the initial depth velocity model that consists of layered model-units containing continuous velocity fields (iteration 1 PSDM, see Section 2.3). A sufficient number of picked horizons (see WB, P, 0, E0, TB, BB, and E1 in Table 2) as well as gridded velocity functions calculated from PSTM (Fig. 6b) were used as the starting point for depth-model building. Here, special attention should be paid to unwanted velocity anomalies. If observed, severe smoothing of velocities must be performed. The initial model was weighted by calibrated sonic logs. As with time model building, the parameter $\delta$ and the velocity trend $V_{0}(z)$ are major factors in the misfit between seismic and well-log velocities. Regarding the choice of appropriate time-to-depth mapping, vertical stretching was found to be a poor approximation due to laterally varying high-velocity intervals. Accurate event positioning that accounts for ray bending at layer interfaces was achieved during migration velocity analysis discussed below.

Depth velocity updates employed traditional image gather analysis as prescribed in Section 2.3 (iterations 2 and 3 PSDM). We followed a basic trial-and-error approach where we tested different velocity perturbations $\Delta V(x, z)$ and evaluated the results based on the changes in the offset migrated domain. Namely, the velocity model is perturbed by a percentage to select the velocity change that best flattens the image gather. We worked to keep the depth interval velocities close to the well velocities in order to minimize time-to-depth conversion errors. It appears that moderate perturbations of the parameter $\delta$ allowed us to create a depth-migrated section with accurate reflector depths. Besides, the piecewise constant $V_{P} / V_{S}$ ratio was scanned until we achieved the best possible flatness of the P-to-S-to-P image gathers along the key horizons listed in Table 2. Recall that this velocity updating process requires pre-processed shot gathers representing output of GDRT wavefield separation (Section 2.1). In this study, it was possible to isolate and migrate symmetric PP and PSPPSP (S travelling inside basalts) wave modes based on the decoupled PSDM workflow (Section 2.3). Advocating a symmetric ray path permits recording of $\mathrm{C} 2$ arrivals by standard towed cable.

We used the velocity model from the above velocity update (Fig. 7) for the final depth migration, and the traveltimes were calculated using the first-arrival ray tracing method augmented with the turning wave option (no evanescent waves). The iterative method of migration and velocity updating was repeated until three conditions were met: an acceptable depth image was observed consistent with available a priori information; focusing energy and overall signal-to-noise ratio were optimised; and primary reflections were flattened on associated image gathers.

To evaluate the benefits contributed by the GDRT wavefield separation, we compare the image gathers obtained from 


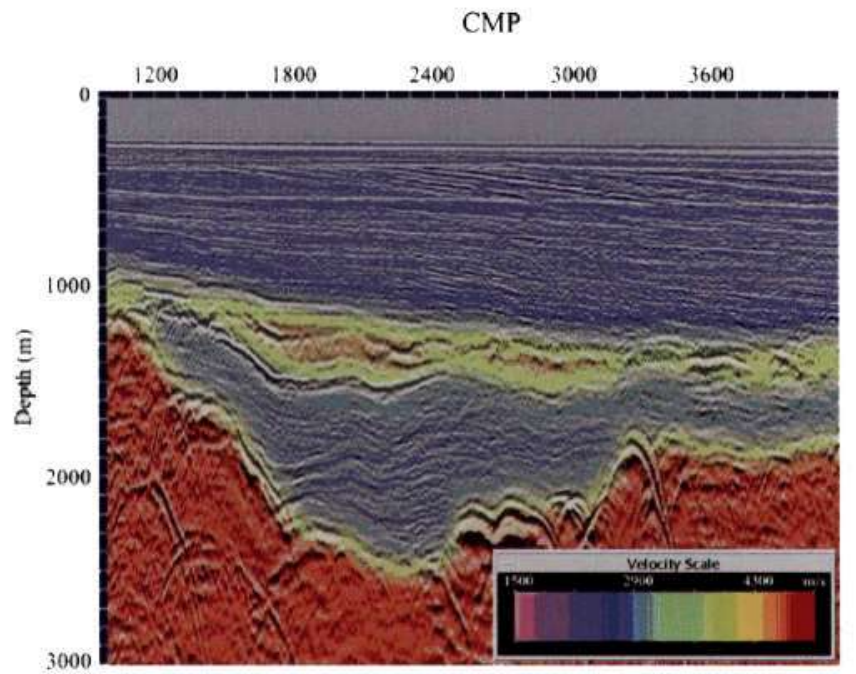

Fig. 7. Depth-domain interval velocity grid model (in $\mathrm{m} / \mathrm{s}$ ) served as the velocity input to PSDM. The velocity model is overlain on the stacked unmigrated section within the CMP range of interest. Principal boundaries of the velocity model are seen. Short-wavelength velocity variations are negligible due to severe smoothness constraints used. See Fig. 2 a for geological legend.

prestack data before and after GDRT for the same CMP location (cf. Figs $8 \mathrm{a}$ and $8 \mathrm{~b}$ ). Both were generated with the velocity model in Fig. 7. Clearly, there are significant differences between the image gathers in Figs $8 \mathrm{a}$ and $8 \mathrm{~b}$. Focusing energy remains diffuse in Fig. 8 a, making depth model updating more challenging. This diffusion is interpreted to be the result of coherent noise in input prestack data. After the noise was suppressed during pre-processing, the revised image gather in
Fig. 8b appears to be generally better focused than in Fig. 8a, indicating that reflected events are properly placed in the offset-depth migrated domain.

After several iterations, the PSDM process yields the depth image in Fig. 9. The overall conclusion is that this velocity model is sufficiently accurate because the image gather in Fig. $8 \mathrm{~b}$ has negligible residual moveout. The depth image in Fig. 9 is summation of the PP image and C2-path image after proper least-squares normalization or spectral matching (White, 1980). It shows good event continuity and high resolution in the zone of interest (enclosed by a dashed box in Fig. 8), allowing more accurate interpretation of the structure above E1, particularly seismic events associated with the BB horizon and reflections beneath this horizon. Individual volcanic units are clearly imaged by high-amplitude events; amplitudes of events adjacent to intrusions locally decrease, probably reflecting increased crack density (Planke et al., 1999). Also, there exists a reasonable correlation between the intra- and sub-basalt structural discontinuities (possibly, a set of sub-vertical faults related to cooling basalt fractures) observed in Fig. 9. Within the target zone $(992<C D P<3332$ and $z \sim 1000-2000 \mathrm{~m})$, the image provides an additional level of detail, giving greater confidence (i.e., lowering risk) in the structural interpretation of the complex HVL structure and sub-basalt reflections. There are a number of (short, coherent) slightly dipping events between the horizons TB and E1. It is not known that these events are truly sub-horizontal or if they are from out of the plane. Below the depth level of $2200 \mathrm{~m}$, imaging is more problematic since the section is dominated by multiples and other coherent noise (Fig. 4) and chaotic energy.
Fig. 8. Example common image gather (CMP = 2699) resulting from depth migration of prestack CMP data $(a)$ before and $(b)$ after iterative GDRT signat entrancement. Identical trace scaling (no automatic gain control) was applied to both gathers. For the sake of comparison with time sections, the vertical axis was transformed from the depth domain into the vertical time $\left(T_{0}\right)$ domain. We insert additional zero traces into the wiggle-trace panel for display purposes only. Overburden and basalt layers are constrained by sonic logs in Figure $2 a$ and time-migrated data in) Figure 6b. Prominent HVL reflections beneath the TB horizon are represented by events $E 1$ (sill reflection) and E2 (basement reflection). The latter is not seen due to noise. Other events are explained in Table 2. Panel (a) shows a significant positive residual moveout (RMO) indicating a general velocity overestimate due to improperly migrated coherent noise. In panel (b). RMO is largely removed due to effective noise attenuation applied.
$\operatorname{AOFFSET}(\mathrm{m})$

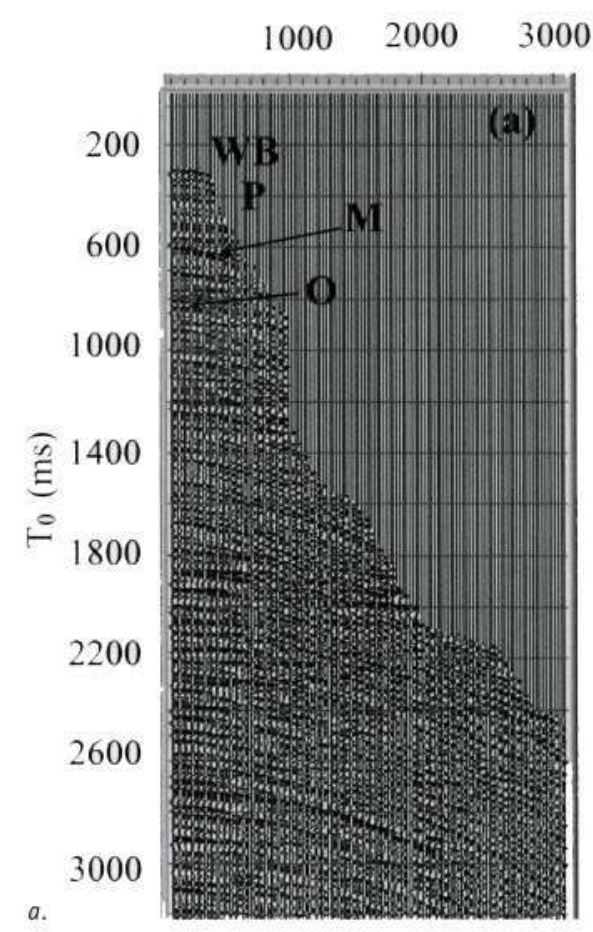

$\operatorname{AOFFSET}(\mathrm{m})$

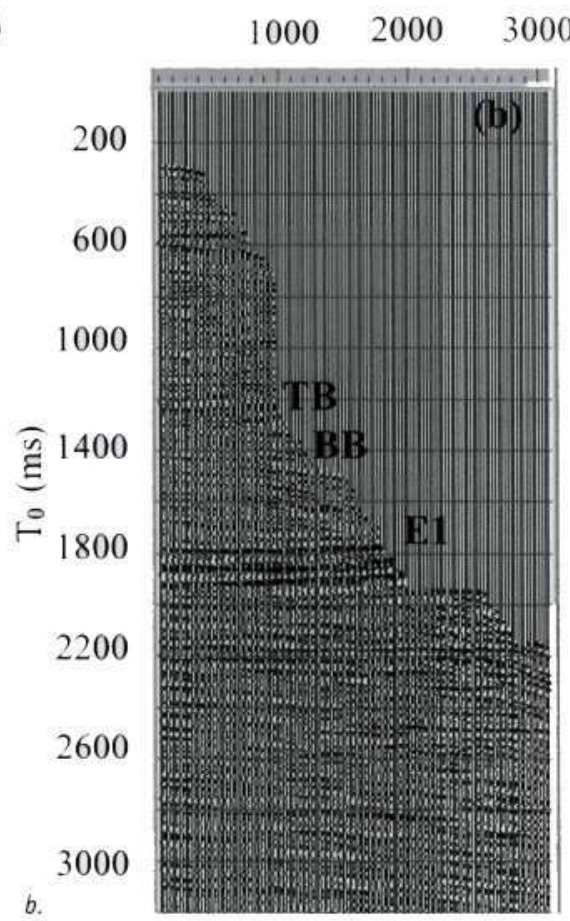




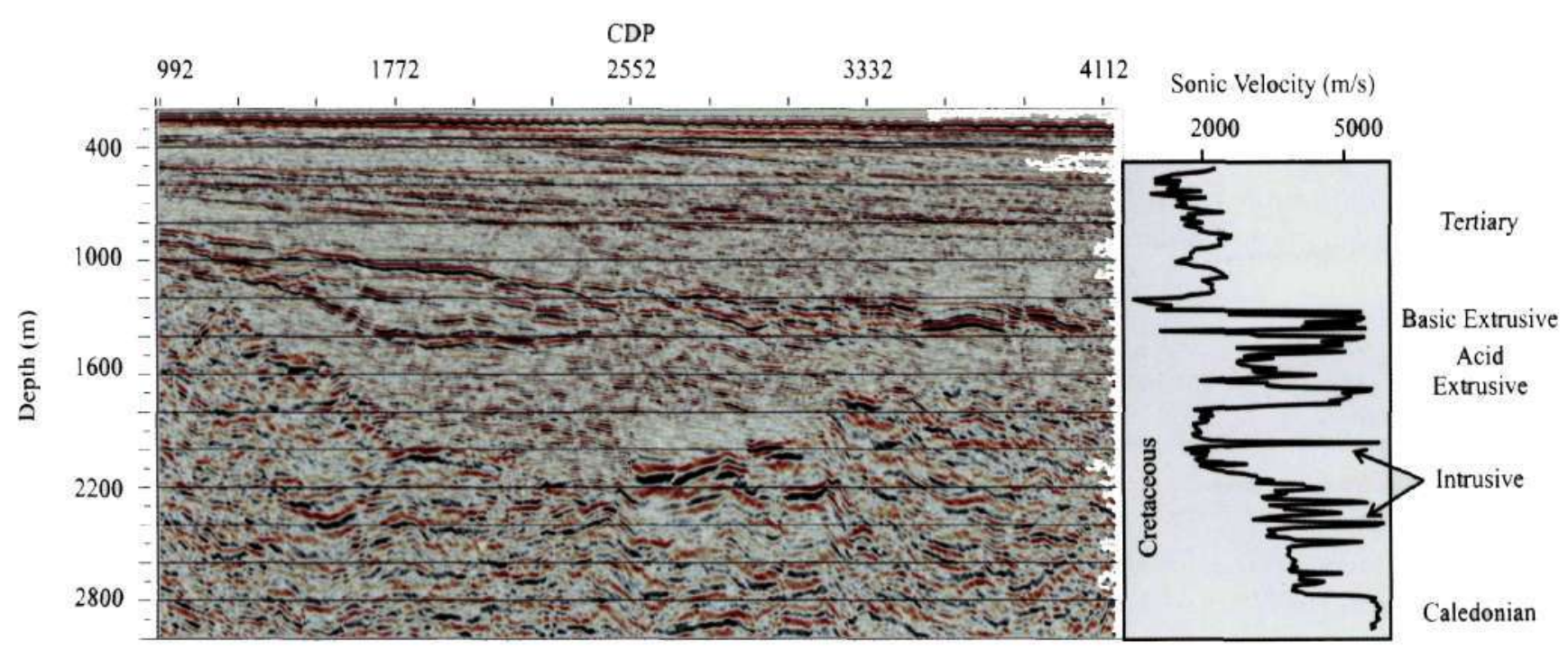

Fig. 9. The final prestack depth-migrated section over the prospect area. Event-based migration was performed after GDRT fittering, trial prestack time migration and migration velocity analysis. This section was generated with the reference velocity model shown in Fig. 7. The sonic velocity curve (fig. $2 a$ ) tied to seismic horizons is attached for lithology discrimination and to support identification of seismic events. Interpretation (IB/BB) is based on horizons derived from prestack time migration (Fig. 6b).

\subsection{Concluding Remarks}

The depth migration results for PP and converted wave modes observed on our data have led to the improved focusing on the final PSDM section in Fig. 9. To minimize potential destructive interference from different frequency content and kinematics of PP and converted wave modes, we applied the GDRT wavefield separation to input gathers. We also leveraged and stacked multi-mode images to reduce spatial ambiguity, typically observed as isochrone noise on individual CIGs due to remaining cross-talk energy. The image in Fig. 9 has shown that weak sub-basalt events now can be clearly interpreted as a series of horizons with faults and pinch-outs. Continuous horizons were confirmed by available well information in Fig. 2. In contrast, sub-basalt PP reflections in Fig. 6 have been poorly focused, and the structure above the event E1 is difficult to interpret. Another technical success in Fig. 9 was the perfect match between depth of seismic events and depth of well tops. The accuracy of well tie was within $15 \mathrm{~m}$. Effective Thomsen's (1986) anisotropy and partial coherence matching of images (White, 1980) have played an important role here. Finally, note that both time and depth imaging steps do not rely on a hyperbolic (NMO) approach and other shortcuts associated with conventional time processing (Yilmaz, 2001).

\section{Discussion}

We have presented an imaging workflow that offers the flexibility to migrate a seismic dataset acquired with standard towed cable from the North of Shetland, UK. The critical steps, with respect to the improvements obtained, are the GDRT filtering of prestack gathers, the construction of the velocity model calibrated by sonic logs, and time/depth migration focusing analysis followed by PSDM. The method has the specific advantage that it enables us to enhance individual events associated with HVLs. We choose the model-based methodology described here and assume that a priori information is available to generate sufficiently accurate velocity estimates. The joint analysis of $\log$ and seismic data demonstrates the ability of the two methods to address imaging issues. We produce a set of image gathers that verify the correctness of the velocity model. We foresee that the best results will be obtained for long-offset seismic profiles because the signal-tonoise ratio of PP reflections and mode conversions at large angles of incidence is better than that of near-offset reflections. Even so, results of this study provide the necessary fast track information to accurately map the radially-dipping structure of the Erlend pluton with consistent intra-basalt reflections. The final image is also capable of imaging subtle features of the surrounding sediment structure beneath the base of basalt.

\section{Acknowledgements}

The first phase of this project (2000-2004) was supported by the EC-funded research project SIMBA coordinated by Total. The second phase $(2004$ - 2005) was funded by the following sponsors of the British Geological Survey (BGS/NERC): $B G$, BGP, BP, Chevron, ConocoPhillips, CNPC, ENI-Agip, ExxonMobil, GX Technology, Kerr-McGee, Landmark, Marathon, Norsk Hydro, PDVSA, Shell, Schlumberger, Total, and Veritas DGC. It is published with the permission of the BGS, SIMBA partners and industrial sponsors. We are grateful to Norsk Hydro for agreeing to release seismic data. Fugro Robertson Ltd. prepared well log 
data for us in accordance with the UK Trade Partners agreement. We acknowledge the administrative support of Paul Williamson (Total), Colin MacBeth (Heriot-Watt University) and Xiang-Yang Li (BGS). We also thank Phil Christie (Schlumberger) and an anonymous reviewer of the paper. Their comments have been very helpful in improving the clarity and readability of the paper.

\section{References}

Barzaghi, L., Calcagni, M., Passolunghi, M. \& Sandroni, S., 2002, Faeroe sub-basalt seismic imaging: a new iterative time processing approach. First Break 20: 611-617.

Beylkin, G., 1982. Generalized Radon transform and its application. PhD thesis, New York University (New York).

Castle, R.J., 1994. A theory of normal moveout. Geophysics 59: 983-999.

Christie, P., Gollifer, I. \& Cowper, D., 2002. Borehole seismic results from the Lopra deepening project. Journal of Conference Abstracts 7(2): 138-139.

Dillon, P.B., Ahmed, H. \& Roberts, T., 1988. Migration of mixed mode VSP wavefields. Geophysical Prospecting 36: 825-846.

Droujinine *, A., 2003. Decoupled elastic prestack depth migration. Journal of Applied Geophysics 54: 369-389.

Droujinine, A., 2005. The attribute based generalized discrete Radon transform. Journal of Seismic Exploration 14: 155-196.

Fruehn, J., Fliedner, M.M. \& White, R.S., 2001. Integrated wide-angle and near-vertical sub-bsalt study using large-aperture seismic data from the Faeroe-Shetland region. Geophysics 66: 1340-1348.

Gatliff, R.W., Hitchen, K., Ritchie, J.D. \& Smythe, D.K., 1984. Internal structure of the Erlend Tertiary volcanic complex, north of Shetland, revealed by seismic reflections. Journal of the Geological Society 141: 555-562.

Hawkins, K., Leggott, R., Williams, G. \& Kat, H., 2001. Addressing anisotropy in 3-D prestack depth migration: A case study from the Southern North Sea. The Leading Edge 20: 528-535.

Jolley, D.W. \& Bell, B.R., 2002. Genesis and age of the Erlend Volcano, NE Atlantic Margin: Geological Society, London, Special Publications 197: 95-110.

Jones, I.F., 2003. A review of 3D preSDM velocity model building techniques. First Break 21: 45-58.

Kuo, J.T. \& Dai, T.-F., 1984. Kirchhoff elastic wave migration for the case of noncoincident source and receiver. Geophysics 49: 1223-1238.

Lambaré, G., Herrmann, P., Guillaume, P., Zimine, S., Wolfarth, S., Hermant, O. \& Butt, S., 2007. From time to depth imaging with 'Beyond Dix'. First Break 25: 71-76.

Maresh, J.\& White, R.S., 2005. Seeing through a glass, darkly: strategies for imaging through basalt. First Break 23: 27-33.

Martini, F. \& Bean, C.J., 2002. Interface scattering versus body scattering in sub-basalt imaging and application of prestack wave equation datuming. Geophysics 67: 1593-1601.

Ogilvie, J.S., Crompton, R. \& Hardy, N.M., 2001. Characterization of volcanic units using detailed velocity analysis in the Atlantic Margin, West of Shetlands, United Kingdom. The Leading Edge 20(1): 34-50.

Planke, S., Alvestad, E. \& Eldholm, O., 1999. Seismic characteristics of basaltic extrusive and intrusive rocks. The Leading Edge 18(3): 342-348.
Purnell, G.W., 1992. Imaging beneath a high-velocity layer using converted waves. Geophysics 57: 1444-1452.

Robein, E. \& Hanitzsch, C., 2001. Benefits of pre-stack time migration in model building: a case history in the South Caspian Sea. First Break 19: 183-189.

Spitzer, R., White, R.S. \& Christie, P.A.F., 2003. Enhancing sub-basalt reflections using parabolic $\tau$ - $p$ transformation. The Leading Edge 22(12): 1184-1201.

Stoker, M.S., Hitchen, K. \& Graham, C.C., 1993. The geology of the Hebrides and West Shetland shelves, and adjacent deep-water areas. HMSO (London).

Takahashi, T., 1995. Prestack migration using arrival angle information. Geophysics 60: 154-163.

Tatham, R.H., Goolsbee, D.V., Massell, W.F. \& Nelson, H.R., 1983. Seismic shear wave observations in a physical model experiment. Geophysics 48: 688-701.

Thomsen, L., 1986. Weak elastic anisotropy. Geophysics 51: 1954-1966.

Thomsen, L., 1999. Converted-wave reflection seismology over inhomogeneous, anisotropic media. Geophysics 64: 678-690.

Van der Baan, M., Kerrane, T., Kendall, J.-M. \& Taylor, N., 2003. Imaging sub-basalt structures using locally converted waves. First Break 21: 29-36.

White, R.E., 1980. Partial coherence matching of synthetic-seismograms with seismic traces. Geophysical Prospecting 28: 333-358.

Yilmaz, 0., 2001. Seismic data analysis. SEG, Tulsa, OK.

* Druzhinin 


\section{Appendix - Estimation of local wavefront attributes}

Let us begin with the CMP local slant stack analysis when the output trace (slant stack) is constructed by shifting and adding the output traces over all CMP locations $x$ and offsets $X$ within a given bin size aperture (typically, $10-20$ traces) surrounding the analysis location $\left\{\tau=t\left(x_{0}, X_{0}\right), x_{0}, X_{0}\right\}$, where $\tau=t(x, X)$ designates the reflection moveout. Indeed, the procedure is to seek the maxima of some local coherency measure function based on semblance, energy power spectrum, covariance matrix, etc. Firstly, for each (reference) CMP position $x_{0}$ and the (reference) offset $X_{0}$ we add CMP traces over a range of offsets along (locally) straight traveltime segments with the slope $F_{0}=\partial_{x} t\left(x_{0}, X_{0}\right)$, i.e. $t\left(x_{0}, X\right) \sim \tau+F_{0}\left(X-X_{0}\right)$. Secondly, for each reference CO section $X=X_{0}$ and the reference CMP position $x_{0}$ we add traces over a range of $x$ along locally straight traveltime segments with the slope $D_{0}=\partial_{x} t\left(x_{0}, X_{0}\right)$, i.e. $t\left(x, X_{0}\right) \sim \tau+D_{0}\left(x-x_{0}\right)$. Those slant-stacked traces $\left\{\tau, F_{0}, D_{0}\right\}$ with a high stack value are saved for later event classification during pre-processing.

Similarly, the common-shot (CS) or common-receiver (CR) local Radon-attribute analysis (Droujinine, 2005) is essentially a beamforming method that employs a procedure of delayand-sum local slant stack processing to steer a local parabolic beam

$$
t\left(x_{0}, X\right) \sim \tau+P\left(X_{0}\right)\left(X-X_{0}\right)+Q\left(X_{0}\right)\left(X-X_{0}\right)^{2}
$$

with the coefficients

$$
P(X)=\frac{\partial t(X)}{\partial X} \text { and } Q(X)=\frac{1}{2} \frac{\partial^{2} t(X)}{\partial X^{2}}
$$

in a particular direction over a range of offsets in the vicinity of $X=X_{0}$. In this case, $x_{0}$ is the $\mathrm{x}$-coordinate of a reference point in the proximity of the observation (source or receiver) point. Firstly, we estimate the local linear moveout

$$
t\left(x_{0}, X\right)-\tau=P\left(X_{0}\right)\left(X-X_{0}\right)
$$

wherever the steering beam half-width aperture is sufficiently small as $X \rightarrow X_{0}$ (typically, a five-to-ten-trace beam aperture is sufficient). Secondly, the local linear moveout

$$
\Delta t\left(x_{0}, X\right)=t\left(x_{0}, X\right)-\tau-P\left(X_{0}\right)\left(X-X_{0}\right)=Q\left(X_{0}\right) \tilde{X}
$$

in the domain $\left\{\tau, \tilde{X}=\left(X-X_{0}\right)^{2}\right\}$ enables us to estimate the wavefront curvature attribute $Q\left(X_{0}\right)$ if the beam aperture is not small (e.g. a twenty-trace bin size can be used). Thus, we transform original CS or CR traces $\{t, x, X\}$ into a set of beamstacked traces $\left\{\tau, x_{0}, P\left(X_{0}\right), Q\left(X_{0}\right)\right\}$. This transformation needs to be computed for each sample in an input trace.
Thus, the $P-Q$ attribute analysis based on eqs. (A1) - (A3) produces local slant stacks by summing traces over a certain range of spatial variables such as $x$ (location) or $X$ (offset). Specifically, we cross correlate or stack the input traces and translate the cross-correlation lag into the local Radonattributes that can be used to compute velocity and reflection time updates. 